\title{
Alternative subtraction scheme using Nagy Soper dipoles
}

\section{Tania Robens*}

Department of Physics and Astronomy, University of Glasgow, Glasgow G12 8QQ, Scotland, $U K$, and

Institut für Theoretische Physik, RWTH Aachen University, 52056 Aachen, Germany

E-mail: trobens@physics.gla.ac.uk

\section{Cheng Han Chung}

Institut für Theoretische Physik, RWTH Aachen University, 52056 Aachen, Germany

E-mail: chenghan@physik.rwth-aachen.de

We present an alternative subtraction scheme for the treatment of infrared divergences in NLO QCD calculations. In this scheme, the number of transformations is greatly reduced with respect to the standard subtraction scheme by Catani and Seymour. We discuss the general setup of the scheme as well as first applications to NLO processes at hadron and lepton colliders.

TTK-10-07, SFB-CPP-10-10

RADCOR 2009 - 9th International Symposium on Radiative Corrections (Applications of Quantum Field Theory to Phenomenology),

October 25 - 302009

Ascona, Switzerland

\footnotetext{
* Speaker.
} 


\section{Introduction: General structure of subtraction schemes}

In higher order calculations, the cancellation of infrared singularities is generally treated by the introduction of an infinitesimal regulator, eg in the form of a finite mass for the massless gauge boson, or by lowering the dimension $D$ of the respective phase space integrals to $D=4-2 \varepsilon$. This way, the analytic cancellation of the respective divergent terms for fully inclusive variables after phase space integration is straightforward; however, numerical implementations of terms containing small regulators prove to be challenging. In subtraction schemes, this problem is circumvented by the introduction of local counterterms, which mimic the behaviour of the squared real emission matrix elements in the singular regions; adding back the respective one particle integrated counterparts to the virtual contributions results in finite integrands for both real emission and virtual contribution phase space. Symbolically, this is given by

$$
\sigma^{\mathrm{NLO}}=\sigma^{\text {Born }}+\sigma^{\text {virt }}+\sigma^{\text {real }}=\sigma^{\text {Born }}+\sigma^{\text {virt }}+\sigma^{\widetilde{A}}+\sigma^{\text {real }}-\sigma^{A},
$$

where

$$
\begin{aligned}
\sigma^{\text {Born }}+\sigma^{\text {virt }}+\sigma^{\widetilde{A}} & =\int d \Gamma_{m}\left[\left|\mathscr{M}_{\text {Born }}\right|^{2}+2 \operatorname{Re}\left(\mathscr{M}_{\text {Born }} \mathscr{M}_{\mathrm{virt}}^{*}\right)+\sum_{\mathrm{i}} \mathscr{V}_{\mathrm{i}}\left|\mathscr{M}_{\text {Born }}\right|^{2}\right] \\
\sigma^{\text {real }}-\sigma^{A} & =\int d \Gamma_{m+1}\left[\left|\mathscr{M}_{\text {real }}\right|^{2}-\sum_{i} D_{i}\left|\mathscr{M}_{\text {Born }}\right|^{2}\right]
\end{aligned}
$$

are the respective $m, m+1$ phase space contributions to the total NLO cross section ${ }^{1}$. Convolution with jet functions then allows to define differential quanities and guarantees infrared safety of the respective Born contribution. In eq (1.2), the sum goes over all local counterterms needed to match the complete singularity structure of the real emission contribution. For each singular limit, the real emission matrix element factorizes according to

$$
\left|\mathscr{M}_{\text {real }}\right|^{2}(p) \longrightarrow D\left|\mathscr{M}_{\text {Born }}\right|^{2}(\tilde{p}),
$$

where $D$ denotes the dipole containing the respective singularity structure. As $\mathscr{M}_{\text {real }}$ and $\mathscr{M}_{\text {Born }}$ live in different phase spaces, a mapping of the respective momenta from $m+1$ to $m$ particle phase space needs to be introduced, which is defined by a mapping function $F_{\text {map }}$ according to $\tilde{p}=F_{\text {map }}(p)$. While the complete singularity structure of the limit considered is contained in $D$, both $D$ and $\mathscr{M}_{\text {Born }}$ can depend on the leftover nonsingular parameters of phase space. $D_{i}$ and $\mathscr{V}_{i}$ are related by $\mathscr{V}_{i}=\mu^{2 \varepsilon} \int d \xi D_{i}$, where the integration measure $d \xi$ is an effective one particle integral. Summarizing, any subtraction scheme needs to fulfill the following requirements:

- definition of subtraction terms $D_{i}$ which, following eq (1.3), one by one mimic the behaviour of the real emission matrix element in each singular region such that their sum contains the complete singularity structure of the process,

- definition of a mapping $F_{\text {map }}$ which guarantees total energy momentum conservation as well as onshellness of all external particles both before and after the mapping,

\footnotetext{
${ }^{1}$ For hadronic initial states, an additional collinear counterterm $\sigma^{C}$ needs to be added in $m$ particle phase space, which accounts for contributions already contained in the NLO PDFs.
} 
- integration measure $d \xi$, with a "smart" choice of variables providing optimal singularity structure parametrization.

In the following, we will discuss this for our specific scheme, comparing with [1] where appropriate.

\section{Scheme setup}

In the scheme discussed in this report, the NLO subtraction terms are derived from the splitting functions introduced in [2], and the $m+1$ to $m$ phase space mappings needed correspond to the inverse of the respective shower $m$ to $m+1$ mappings. In the following, we will denote the $m+1$ phase space four vectors by $\hat{p}_{1}, \hat{p}_{2}, \ldots$ and $m$ phase space four vectors by $p_{1}, p_{2}, \ldots$ Indices $a, b$ will denote initial state particles; in $m+1$ phase space, $\hat{p}_{i}$ is the emitter, $\hat{p}_{j}$ the emitted particle, and $\hat{p}_{k}$ the spectator ${ }^{2}$. By default, for initial state emissions we set $\hat{p}_{i}=\hat{p}_{a}$ in all general expressions below. Equally, we restrict our expressions to subtractions on the parton level and to the massless case; details on convolution with PDFs are given in [2], and the extension to massive particles is in the line of future work.

\subsection{Momentum mapping and integration measure: Initial state}

For the initial state, the mapping from the $m+1$ to the $m$ particle phase space is given by

$$
p_{a}=\left(1-\frac{\hat{p}_{j} \cdot \hat{Q}}{\hat{p}_{a} \cdot \hat{p}_{b}}\right) \hat{p}_{a}, p_{l}=\Lambda(K, \widehat{K}) \hat{p}_{l}, p_{b}=\hat{p}_{b},
$$

where the index $l$ goes over all final state particles in the $m$ particle phase space and with

$$
\Lambda(K, \widehat{K})=g-\frac{2(K+\widehat{K})(K+\widehat{K})}{(K+\widehat{K})^{2}}+\frac{2 K \widehat{K}}{\widehat{K}^{2}},
$$

where $K=p_{a}+p_{b}, \hat{Q}=\hat{p}_{a}+\hat{p}_{b}, \widehat{K}=\hat{Q}-\hat{p}_{j}$. The phase space factorizes according to

$$
\left[d\{\hat{p}, \hat{f}\}_{m+1}\right] g\left(\{\hat{p}, \hat{f}\}_{m+1}\right)=\left[d\{p, f\}_{m}\right] d \xi_{p} g\left(\{\hat{p}, \hat{f}\}_{m+1}\right),
$$

where $f$ denotes the flavour and with the D-dimensional integration measure

$$
d \xi_{p}=\frac{d^{D} \hat{p}_{j}}{(2 \pi)^{D-1}} \delta_{+}\left(\hat{p}_{j}^{2}\right) .
$$

\subsection{Momentum mapping and integration measure: Final state}

For final state splittings, the initial state momenta remain unchanged: $p_{a}=\hat{p}_{a}, p_{b}=\hat{p}_{b}$. The mapping uses all non-emitting particles as one spectator for momentum redistribution. We introduce the additional variables

$$
\begin{aligned}
& P=\hat{p}_{i}+\hat{p}_{j}, Q=\hat{p}_{a}+\hat{p}_{b}, \\
& y=\frac{P^{2}}{2 P \cdot Q-P^{2}}, a=\frac{Q^{2}}{2 P \cdot Q-P^{2}}, \lambda=\sqrt{(1+y)^{2}-4 a y} .
\end{aligned}
$$

\footnotetext{
${ }^{2}$ In contrast to [1], in our case a spectator only needs to be specified if $\hat{p}_{j}$ denotes a gluon.
} 
The emitting particle is mapped according to

$$
p_{i}=\frac{P}{\lambda}-\frac{1-\lambda+y}{2 \lambda a} Q .
$$

All non-emitting final state particles are mapped using the Lorentz transformation as in eq. (2.2), where now $K=Q-p_{i}, \widehat{K}=Q-P$. Especially, this means that the total number of mappings needed for a $N$-jet final state scales as $\frac{N^{2}}{2}$, which reduces scaling ${ }^{3}$ with respect to [1] by a factor $N$. The phase space factorization takes a similar form as in the initial state splitting, ie we have again

$$
\left[d\{\hat{p}, \hat{f}\}_{m+1}\right] g\left(\{\hat{p}, \hat{f}\}_{m+1}\right)=\left[d\{p, f\}_{m}\right] d \xi_{p} g\left(\{\hat{p}, \hat{f}\}_{m+1}\right)
$$

where now

$$
\begin{aligned}
d \xi_{p}= & d y \theta\left(y_{\max }-y\right) \lambda^{D-3} \frac{p_{i} \cdot Q}{\pi} \frac{d^{D} \hat{p}_{i}}{(2 \pi)^{D-1}} \delta_{+}\left(\hat{p}_{i}^{2}\right) \frac{d^{D} \hat{p}_{j}}{(2 \pi)^{D-1}} \delta_{+}\left(\hat{p}_{j}^{2}\right) \\
& \times(2 \pi)^{D} \delta^{(D)}\left(P-\lambda p_{i}-\frac{1-\lambda+y}{2 a} Q\right) .
\end{aligned}
$$

$y_{\max }=(\sqrt{a}-\sqrt{a-1})^{2}$ can directly be derived from total energy momentum conservation.

\subsection{Treatment of interference terms: dipole partitioning functions}

Double poles in splitting functions only arise if the emitted particle is a gluon; in this case, interference terms between different emitters have to be taken into account. In our scheme, we split the collinear and soft parts of the respective spin averaged splitting functions $\bar{W}$ according to [4]

$$
\bar{W}_{i i}-\bar{W}_{i k}=\left(\bar{W}_{i i}-\bar{W}_{i i}^{\mathrm{eik}}\right)+\left(\bar{W}_{i i}^{\mathrm{eik}}-\bar{W}_{i k}\right),
$$

where $\bar{W}_{i i}^{\text {eik }}$ is the spin-averaged eikonal factor. The second part of the above equation can be then expressed in terms of dipole partitioning functions $A_{i k}^{\prime}[5]$

$$
\bar{W}_{i i}^{\mathrm{eik}}-\bar{W}_{i k}=4 \pi \alpha_{s} A_{i k}^{\prime} \frac{-\hat{P}_{i k}^{2}}{\left(\hat{p}_{j} \cdot \hat{p}_{i} \hat{p}_{j} \cdot \hat{p}_{k}\right)^{2}},
$$

where $\hat{P}_{i k}=\hat{p}_{j} \cdot \hat{p}_{i} \hat{p}_{k}-\hat{p}_{j} \cdot \hat{p}_{k} \hat{p}_{i}$. Several choices for $A_{i k}^{\prime}$ have been proposed [5]; all results given here have been obtained using eq (7.12) therein.

\section{Example of integrated splitting function: $g \rightarrow q \bar{q}$ final state splitting}

For our scheme, all collinear as well as singular parts of the soft splitting functions have been tested; a complete list will be given in [6]. In this section, we give the final state $g \rightarrow q \bar{q}$ dipole and the corresponding integrated term as an example, additionally commenting on the limit for $m \rightarrow 2$.

For a $g \rightarrow q \bar{q}$ splitting in the massless case, the spin averaged subtraction term is given by $D_{g q \bar{q}}\left|\mathscr{M}_{\mathrm{Born}}(p)\right|^{2}$, with

$$
D_{g q \bar{q}}=T_{R} \frac{4 \pi \alpha_{s}}{y p_{i} \cdot Q}\left[1-\frac{2 z(1-z)}{1-\varepsilon}\right],
$$

\footnotetext{
${ }^{3}$ The subtraction scheme in [3] has a scaling similar to our scheme.
} 
where we introduced the additional variables $z=\frac{\hat{p}_{j} \tilde{n}}{P \tilde{n}}, \tilde{n}=\frac{1+y+\lambda}{2 \lambda} Q-\frac{a}{\lambda} P$, and all other variables as in eq. (2.5). This subtraction term was derived by squaring the respective final state shower splitting function in [2]. Momentum mapping is done according to eq. (2.6) and the Lorentz transform with the respective expressions for $K, \hat{K}$. For the integrated splitting function, we rewrite the measure (2.8) in terms of the variables introduced above and obtain

$$
d \xi_{p}=\frac{\left(2 p_{i} Q\right)^{1-\varepsilon}}{16 \pi^{2}} \frac{d \Omega_{d-2}}{(2 \pi)^{1-2 \varepsilon}} d z d y \lambda^{1-2 \varepsilon} y^{-\varepsilon}[z(1-z)]^{-\varepsilon} \theta\left(y_{\max }-y\right) \theta[z(1-z)]
$$

which results in

$$
\mathscr{V}=\mu^{2 \varepsilon} \int d \xi_{p} D=T_{R} \frac{\alpha_{s}}{2 \pi} \frac{1}{\Gamma(1-\varepsilon)}\left(\frac{2 \pi \mu^{2}}{p_{i} Q}\right)^{\varepsilon}\left[-\frac{2}{3 \varepsilon}-\frac{16}{9}+\frac{2}{3}[(a-1) \ln (a-1)-a \ln a]\right] .
$$

As expected, for $m=2$ the above expressions as well as the mapping completely reduce to the result in [1].

We want to comment that in our scheme, the most complicated expressions stem from the integration of the interference terms as in eq. (2.10). As all final state particles are mapped using the Lorentz transform $\Lambda$, the finite parts of the respective subtraction functions need to be evaluated numerically; details will be given in [6].

\section{First results}

As an example, we give the analytic result of our splitting functions when applied to dijet production at lepton colliders, as well as a numerical comparison for Drell-Yan process at NLO using [1] as well as the scheme proposed in this writeup. We additionally tested the scheme for Higgs production at hadron colliders and decay; the respective calculations will be presented elsewhere.

\subsection{Dijet production at lepton colliders}

For dijet production at lepton colliders, the final state squared splitting function $D=D_{q q g}$ is needed. We denote the four-momenta of the outgoing partons in this process with $\hat{p}_{1}(q), \hat{p}_{2}(\bar{q})$, and $\hat{p}_{3}(g)$. The unintegrated dipole subtraction term for emission from $\hat{p}_{1}$ is then given by

$$
\begin{aligned}
D & =\frac{8 \pi \alpha_{s}}{\hat{Q}^{2}} C_{F}\left\{\left(\frac{1}{x_{2}}\right)\left[2\left(\frac{x_{1}}{2-x_{1}-x_{2}}-\frac{1-x_{2}}{\left(2-x_{1}-x_{2}\right)^{2}}\right)+\frac{1-x_{1}}{1-x_{2}}\right]\right. \\
& \left.+2\left(\frac{x_{1}+x_{2}-1}{1-x_{2}}\right) \frac{x_{1}}{\left(1-x_{1}\right) x_{1}+\left(1-x_{2}\right) x_{2}}\right\}
\end{aligned}
$$

with $x_{n}=\frac{2 \hat{p}_{n} \hat{Q}}{\hat{Q}^{2}}$. The respective integrated averaged splitting function is

$$
\mathscr{V}=\frac{\alpha_{s}}{2 \pi} C_{F} \frac{1}{\Gamma(1-\varepsilon)}\left(\frac{4 \pi \mu^{2}}{Q^{2}}\right)^{\varepsilon}\left[\frac{1}{\varepsilon^{2}}+\frac{3}{2 \varepsilon}-1+\frac{\pi^{2}}{6}\right] .
$$

Combining the above splitting functions for both emitters with the Born, real emission, and virtual matrix elements and integrating over phase space, we obtain the standard result

$$
\sigma^{N L O}=\sigma^{N L O\{2\}}+\sigma^{N L O\{3\}}=\frac{\alpha_{s}}{2 \pi} C_{F}\left[\left(-10+\frac{4}{3} \pi^{2}\right)+\left(\frac{23}{2}-\frac{4}{3} \pi^{2}\right)\right] \sigma^{L O}=\frac{3}{4} \frac{\alpha_{s}}{\pi} C_{F} \sigma^{L O} .
$$




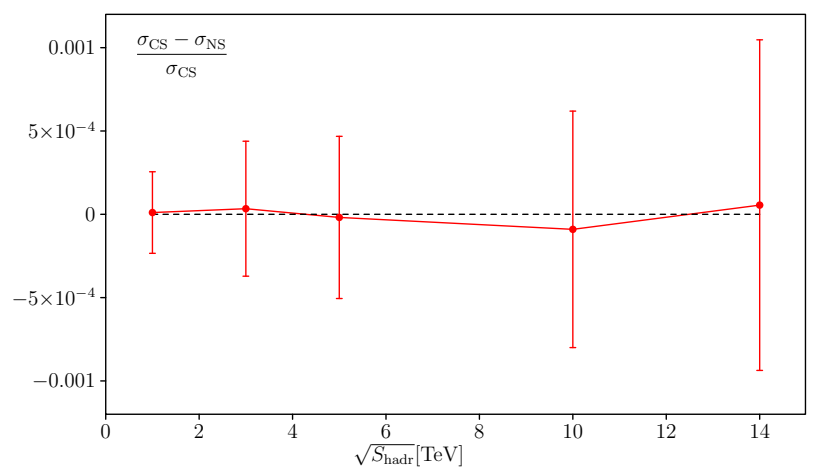

Figure 1: Relative difference between NLO corrections to single W production using Catani Seymour and Nagy Soper dipoles respectively, as a function of the hardonic cm energy. The results agree on sub-permil level, shown are the numerical integration errors.

\subsection{Drell-Yan production}

We calculated single $W$ production for a $p p$ initial state at NLO, using both the scheme in [1] as well as our scheme, including PDFs and varying the hadronic cm energy of the process. We here only show the numerical result for this process. Figure 1 plots the relative difference between the two implemented schemes. We see that the numerical differences are on the permill level and consistent with zero.

\section{Summary and Outlook}

In this report, we introduce a subtraction scheme which reduces the number of mappings in the real emission part of an NLO calculations by a factor $N_{\text {jets }}$ with respect to the scheme suggested in [1]. We explained the setup as well as phase space mapping, and presented first results for an integrated splitting function as well as applications for simple processes. A complete listing of all integrated splitting functions as well as further examples will be given in [6].

\section{References}

[1] S. Catani and M. H. Seymour, Nucl. Phys. B 485, 291 (1997) [Erratum-ibid. B 510, 503 (1998)] [arXiv:hep-ph/9605323].

[2] Z. Nagy and D. E. Soper, JHEP 0709, 114 (2007) [arXiv:0706.0017 [hep-ph]].

[3] S. Frixione, Z. Kunszt and A. Signer, Nucl. Phys. B 467, 399 (1996) [arXiv:hep-ph/9512328].

[4] Z. Nagy and D. E. Soper, JHEP 0803, 030 (2008) [arXiv:0801.1917 [hep-ph]].

[5] Z. Nagy and D. E. Soper, JHEP 0807, 025 (2008) [arXiv:0805.0216 [hep-ph]].

[6] C. Chung, M. Krämer, Z. Nagy, T. Robens and D. E. Soper, in preparation 